\title{
On spectrum of ILW hierarchy in conformal field theory II: coset CFT's
}

\author{
M.N. Alfimov ${ }^{a, b, c, d}$ and A.V. Litvinov ${ }^{e, f}$ \\ ${ }^{a}$ LPT, Ecole Normale Superieure, \\ 75005 Paris, France \\ ${ }^{b}$ Insitut de Physique Theorique, CEA Saclay, \\ 91191 Gif-sur-Yvette Cedex, France \\ ${ }^{c}$ P.N. Lebedev Physical Institute, \\ 119991 Moscow, Russia \\ ${ }^{d}$ Moscow Institute of Physics and Technology, \\ 141700 Dolgoprudny, Russia \\ ${ }^{e}$ Landau Institute for Theoretical Physics, \\ 142432 Chernogolovka, Russia \\ ${ }^{f}$ NHETC, Department of Physics and Astronomy, Rutgers University, \\ Piscataway, NJ 08855-0849, U.S.A. \\ E-mail: alfimov.mikhail@gmail.com, litvinov@itp.ac.ru
}

ABSTRACT: We study integrable structure of the coset conformal field theory and define the system of Integrals of Motion which depends on external parameters. This system can be viewed as a quantization of the ILW type hierarchy. We propose a set of Bethe anzatz equations for its spectrum.

KeYwords: Conformal and W Symmetry, Bethe Ansatz, Integrable Field Theories

ARXIV EPRINT: 1411.3313 


\section{Contents}

1 Introduction 1

2 The case $p=1$ and generic $r: \mathrm{W}$-algebras 4

3 The case $r=1$ and generic $p: \widehat{\mathfrak{g l}}(p)_{1}$ algebra 6

4 The case $r=p=2$ : quantum SUSY KdV system $\quad 7$

$\begin{array}{llr}5 & \text { Conclusions } & 9\end{array}$

\section{Introduction}

This paper is a direct continuation of [1] where the set of Bethe anzatz equations for the spectrum of Integrals of Motion (IM) in Conformal Field Theory (CFT) was proposed. Here we consider more general class of CFT's defined by GKO coset construction [2]

$$
\mathcal{M}(r, p, n)=\frac{\widehat{\mathfrak{s l}}(r)_{p} \times \widehat{\mathfrak{s l}}(r)_{n-p}}{\widehat{\mathfrak{s l}}(r)_{n}} .
$$

This set of CFT's is unitary for non-negative integer values of the parameters $p$ and $n-p$ and includes the $W$ unitary minimal models $\mathcal{W}_{\mathcal{A}_{r}}(k)=\mathcal{M}(r, 1, k-r)$ as a particular case.

It is well known [3] that among primary fields of the model $\mathcal{M}(r, p, n)$ there is a special one which defines an integrable perturbation. It has conformal dimension $\Delta=\frac{n}{n+r}$ and corresponds by GKO construction to the branching of the product of two vacuum representations into adjoint one. This operator is a complete analog of $\Phi_{1,3}$ operator in minimal models. According to [4] such an integrable perturbation defines an infinite set of commuting operators called local Integrals of Motion. In this paper we study the problem of computation of their common spectrum.

The coset CFT's (1.1) admit some chiral algebra description. Typically, it involves currents of fractional spins with non-abelian braiding which makes it difficult to analyze such models. In some cases the description simplifies and the chiral algebra reduces to known algebras. For example we get $\mathrm{W}_{r}$ algebra [5-7] for $p=1$, NSR algebra for $r=p=2$ and spin- $\frac{4}{3}$ parafermion algebra [8] for $r=2, p=4$. For us it will be important that the chiral algebra description of the coset models (1.1) exists and depends smoothly on the parameter $n$ which we parameterize as

$$
n=\frac{p}{1+b^{2}}-r
$$


The new parameter $b$ will be treated below as continuous and we denote the corresponding chiral algebra as $\mathcal{A}(r, p)$. In new notations its central charge can be written as

$$
c=\frac{p\left(r^{2}-1\right)}{p+r}+\frac{r\left(r^{2}-1\right)}{p} Q^{2}, \quad \text { where } Q=b+\frac{1}{b} .
$$

Sometimes it is convenient to think about the algebra $\mathcal{A}(r, p)$ as a symmetry algebra of parafermionic Toda theory [9]. This theory can be realized as a coupled theory of $(r-1)$ component bosonic field $\varphi$ and $\widehat{\mathfrak{s l}}(r)_{p} / \widehat{\mathfrak{u}}(1)^{r-1}$ parafermions [10]. The Lagrangian can be schematically written as follows

$$
\mathcal{L}=\mathcal{L}_{\mathrm{PF}}+\frac{p}{8 \pi}\left(\partial_{a} \varphi\right)^{2}+\mu \sum_{k=1}^{r-1} \Psi_{e_{k}} \bar{\Psi}_{e_{k}} e^{b\left(e_{k}, \varphi\right)},
$$

where $\Psi_{e_{k}}$ is a parafermionic current corresponding to the simple root $e_{k}$ of $\mathfrak{s l}(r), \bar{\Psi}_{e_{k}}$ its complex conjugate and $\mathcal{L}_{\mathrm{PF}}$ is a formal Lagrangian for the parafermionic CFT. In these terms the integrable perturbation operator has the form $\Psi_{e_{0}} \bar{\Psi}_{e_{0}} e^{b\left(e_{0}, \varphi\right)}$, where $e_{0}$ is the lowest root. Mathematically, one can define a system of local IM's as a set of local quantities in $\mathcal{U}(\mathcal{A}(r, p))$ which commute with the chiral parts of all the fields

$$
\int_{\mathcal{C}} \Psi_{e_{k}} \bar{\Psi}_{e_{k}} e^{b\left(e_{k}, \varphi\right)} d x, \quad \text { for } \quad k=0, \ldots, r-1 .
$$

For $p=1$ this system is known to coincide with the quantum KdV type system with Lax operator of order $r$ [11]. This includes quantum KdV system for $r=2$ (related to the Virasoro algebra) and quantum Boussinesq system for $r=3$ (related to the $\mathrm{W}_{3}$ algebra). For generic values of the parameters $r$ and $p$ we call the corresponding integrable system quantum KdV system of type $(r, p)$ or qKdV $(r, p)$.

On the other hand the symmetry algebra of the coset (1.1) appeared recently in the context of AGT relation [12]. Namely, it was conjectured in [13] that $\mathrm{U}(r)$ instanton calculus on the quotient $\mathbb{C}^{2} / \mathbb{Z}_{p}$ corresponds to the extended algebra

$$
\widehat{\mathfrak{g l}}(p)_{r} \times \mathcal{A}(r, p) .
$$

The original conjecture of [13] was further checked in [14-18]. Mathematically, the results of [13] predict the existence of a special basis in the highest weight representation of the algebra $\widehat{\mathfrak{g l}}(p)_{r} \times \mathcal{A}(r, p)$ with remarkable property of factorization of matrix elements. In fact, as was emphasized in $[19,20]$, there are different bases corresponding to different compactifications of the space of instantons on $\mathbb{C}^{2} / \mathbb{Z}_{p}$. For our purposes the so called "colored" basis (see [20] for details) is more suitable. This basis is an eigenbasis for the commutative algebra (Integrals of Motion) inside universal enveloping algebra of $\widehat{\mathfrak{g l}}(p)_{r} \times$ $\mathcal{A}(r, p)$ with remarkably simple spectrum. For example, in the case of $r=1$ we get the spin Calogero-Sutherland integrable system [21], whose eigenfunctions are known as Uglov polynomials [22] (also known as $\mathfrak{g l}(p)$-Jack polynomials). In the case of $r>1$ the corresponding integrable system can be viewed as coupled system of $r$ copies of spin Calogero-Sutherland models. We call this system the generalized Calogero-Sutherland integrable system of the type $(r, p)$ or $\operatorname{CS}(r, p)$. 
In these notes we consider $p$-parametric integrable system which interpolates between $\mathrm{CS}(r, p)$ and $\mathrm{qKdV}(r, p)$. Namely, we state that there exists an infinite commutative family of operators $\mathbf{I}_{k} \in \mathcal{U}\left(\widehat{\mathfrak{g l}}(p)_{r} \times \mathcal{A}(r, p)\right)$ of spins $k=1,2, \ldots$ depending on $p$ additional parameters $\left(q_{1}, \ldots, q_{p}\right)$ which coincides with the $\mathrm{CS}(r, p)$ system for $\left(q_{1}, \ldots, q_{p}\right)=(0, \ldots, 0)$ and degenerates to $\mathrm{qKdV}(r, p)$ in the limit

$$
\left(q_{1}, \ldots, q_{p}\right) \rightarrow(1, \ldots, 1) .
$$

We call this integrable system quantum Intermediate Long Wave system of type $(r, p)$ or qILW $(r, p)$ for shortness. The reason for that is the same as above: for $p=1$ this system is known to be quantum $\mathfrak{g l}(r)$ ILW system.

The main result of our paper is the claim that the common spectrum of $\mathbf{I}_{k} \in \mathcal{U}\left(\widehat{\mathfrak{g l}}(p)_{r} \times\right.$ $\mathcal{A}(r, p))$ is governed by Bethe equations. In order to write down these equations we introduce three functions

$$
\mathbb{S}_{0}(x)=\frac{x+Q}{x-Q}, \quad \mathbb{S}_{+}(x)=\frac{x-b}{x+b^{-1}}, \quad \mathbb{S}_{-}(x)=\frac{x-b^{-1}}{x+b} .
$$

Then, for given $r$ and $p$ let $\left(N_{1}, \ldots, N_{p}\right)$ be a set of non-negative integers and $\mathbb{A}_{k}(x)$ $(k=1, \ldots, p)$ be a set polynomials of degree $d_{k}$ :

$$
\mathbb{A}_{k}(x)=\prod_{j=1}^{d_{k}}\left(x+i P_{j}^{(k)}\right)
$$

such that $\sum_{k=1}^{p} d_{k}=r$ and $\sum_{k=1}^{p} \sum_{j=1}^{d_{k}} P_{j}^{(k)}=0$. All these parameters correspond in a certain way to the representation data of $\widehat{\mathfrak{g l}}(p)_{r} \times \mathcal{A}(r, p)$. Namely, the numbers $\left(d_{1}, \ldots, d_{p}\right)$ parameterize the highest weight of $\widehat{\mathfrak{g l}}(p)_{r}$, the continuous parameters $P_{j}^{(k)}$ correspond to the zero modes of the bosonic field $\varphi$ in (1.4) and the set $\left(N_{1}, \ldots, N_{p}\right)$ corresponds to the level. With each $N_{k}$ one associates a set of variables

$$
x_{j}^{(k)}, \quad j=1, \ldots, N_{k},
$$

where the cyclic symmetry is assumed $x_{j}^{(k)}=x_{j}^{(p+k)}, N_{k}=N_{p+k}$. Then our conjecture states:

Conjecture. The spectrum of $\mathrm{qILW}(r, p)$ is governed by the Bethe anzatz equations

$$
\frac{\mathbb{A}_{k}\left(x_{j}^{(k)}-\frac{Q}{2}\right)}{\mathbb{A}_{k}\left(x_{j}^{(k)}+\frac{Q}{2}\right)} \prod_{i=1}^{N_{k}} \mathbb{S}_{0}\left(x_{j}^{(k)}-x_{i}^{(k)}\right) \prod_{i=1}^{N_{k+1}} \mathbb{S}_{+}\left(x_{j}^{(k)}-x_{i}^{(k+1)}\right) \prod_{i=1}^{N_{k-1}} \mathbb{S}_{-}\left(x_{j}^{(k)}-x_{i}^{(k-1)}\right)=-q_{k},
$$

for $j=1, \ldots, N_{k}, k=1, \ldots, p$.

In order to make our conjecture more precise we need to construct IM's $\mathbf{I}_{k}$ explicitly and establish the relation between their eigenvalues and roots of (1.10). It is hard to do it in a full generality. This is why we consider only particular cases of our conjecture in next sections. Namely, in section 2 we consider the case of $p=1$ and generic $r$ which corresponds to the $\mathrm{W}_{r}$ algebras, in section 3 we consider "orthogonal" direction: $r=1$ 
and generic $p$ corresponding to the free fermion theory $\widehat{\mathfrak{g r}}(p)_{1}$ and finally in section 4 we consider a special case $p=r=2$ which corresponds to the NSR algebra. Details of the generic case will be given in a separate publication.

Let us make a few remarks. First, as explained above, physically interesting situation corresponds to the limit (1.7). In this limit the $\widehat{\mathfrak{g l}}(p)_{r}$ part of the symmetry algebra decouples and the system (1.10) describes spectrum of IM's for the coset CFT (1.1) (qKdV $r, p)$ system). However, for numerical purposes it is convenient to keep the parameters $q_{k}$ (twist parameters) away from criticality. Second, there is a relation between quantum integrable systems of ILW type and quantum cohomology [23-27]. In our approach we do not use this relation. Third, all our statements are of conjectural nature. They are based on explicit calculations for the lower levels. The derivation of Bethe anzatz equations (1.10) from the first principles is an open problem. We discuss this problem as well as other interesting questions in concluding section 5.

\section{The case $p=1$ and generic $r: \mathrm{W}$-algebras}

In this case we have $\widehat{\mathfrak{g l}}(1)_{r} \times \mathcal{A}(r, 1)=\mathrm{H} \times \mathrm{W}_{r}$. The algebra $\mathcal{A}(r, 1)=\mathrm{W}_{r}$ is generated by holomorphic currents $T(z)$ and $W(z)$ of spins 2 and 3. Currents with higher spins from 4 to $r$ appear in the OPE of $W(z)$. We fix $W(z)$ as usual: it is primary field of spin 3 normalized as

$$
W(\xi) W(z)=\frac{c}{3(\xi-z)^{6}}+\ldots
$$

with $c=(r-1)\left(1+r(r+1) Q^{2}\right)$ (see (1.3)). The commutation relations of the components of these currents are rather cumbersome. For $r=3$ they can be found in [6]. The Heisenberg algebra $\mathrm{H}$ is given by commutation relations (we set $a_{0}=0$ for convenience)

$$
\left[a_{m}, a_{n}\right]=m \delta_{m,-n} .
$$

Working with $\mathrm{W}_{r}$ algebras it is convenient to use $\mathfrak{s l}(r)$ Lie algebra notations: $e_{k}$ are the simple roots of $\mathfrak{s l}(r), \omega_{k}$ are the fundamental weights, $h_{k}$ are the weights of fundamental representation and $\rho$ is the Weyl vector. We define the highest weight state $|\mathcal{P}\rangle$ where $\mathcal{P}$ is the $r-1$ component momentum as the state which is "killed" by the positive part of the algebra $\mathrm{H} \times \mathrm{W}_{r}$ and is an eigenstate for its Cartan subalgebra:

$$
a_{n}|\mathcal{P}\rangle=L_{n}|\mathcal{P}\rangle=W_{n}|\mathcal{P}\rangle=\ldots=0 \quad n>0, \quad L_{0}|\mathcal{P}\rangle=\Delta(P)|\mathcal{P}\rangle, \quad W_{0}|\mathcal{P}\rangle=w(\mathcal{P})|\mathcal{P}\rangle, \ldots
$$

Here $L_{n}, W_{n}, \ldots$ are the components of the currents $T(z), W(z), \ldots$ and $(\Delta(\mathcal{P}), w(\mathcal{P}), \ldots)$ are some Weyl invariant functions of $\mathcal{P}$. In particular,

$\Delta(\mathcal{P})=\frac{1}{2}(\mathcal{Q}+i \mathcal{P}, \mathcal{Q}-i \mathcal{P})=\frac{r\left(r^{2}-1\right) Q^{2}}{24}+\frac{1}{2} \sum_{k=1}^{r} P_{k}^{2}, \quad$ here $\quad \mathcal{Q}=Q \rho \quad$ and $\quad P_{k}=\left(\mathcal{P}, h_{k}\right)$.

The highest weight representation $\pi_{\mathcal{P}}$ of the algebra $\mathrm{H} \oplus \mathrm{W}_{r}$ is formed from $|\mathcal{P}\rangle$ by creation operators. If the momentum $\mathcal{P}$ is generic then the corresponding representation is irreducible. The number of states on given level $N$ (the eigenvalue of the operator $L_{0}^{\text {total }}-\Delta$ ) is $\mathfrak{p}(r, N)$, where $\mathfrak{p}(r, N)$ is the number of $r$-partitions of $N$. 
We define the system of IM's $\mathbf{I}_{k} \in \mathcal{U}\left(\mathrm{H} \oplus \mathrm{W}_{r}\right)$ which depends on additional parameter $q_{1}=q$. First two IM's have the form

$$
\begin{aligned}
& \mathbf{I}_{1}=L_{0}+\sum_{k=1}^{\infty} a_{-k} a_{k}-\frac{c+1}{24}, \\
& \mathbf{I}_{2}=\sqrt{\frac{r\left(r^{2}-4\right) Q^{2}}{2}+2(r-2)} W_{0}+2 \sum_{k \neq 0} L_{-k} a_{k}+i r \sqrt{r} Q \sum_{k=1}^{\infty} k \frac{1+q^{k}}{1-q^{k}} a_{-k} a_{k}+\frac{1}{3} \sum_{i+j+k=0} a_{i} a_{j} a_{k} .
\end{aligned}
$$

The remaining integrals $\mathbf{I}_{k}$ can be in principle determined from the condition of mutual commutativity with $\mathbf{I}_{1}$ and $\mathbf{I}_{2}$ (see [1] for expressions for higher IM's for $r=2$ ). Sometimes it is convenient to rewrite IM's as integrals of density functions. For example, we can write $\mathbf{I}_{2}$ as

$\mathbf{I}_{2}=\frac{1}{2 \pi} \int_{0}^{2 \pi}\left(\sqrt{\frac{r\left(r^{2}-4\right) Q^{2}}{2}+2(r-2)} W(x)+2 T(x) J(x)+\frac{i r \sqrt{r} Q}{2} J(x) \mathcal{D} J(x)+\frac{1}{3} J(x)^{3}\right) d x$,

where $J(x)$ is a $\mathrm{U}(1)$ current $J(x)=\sum_{k} a_{k} e^{-i k x}$ and $\mathcal{D}$ is an operator whose Fourier symbol is $k \frac{1+q^{k}}{1-q^{k}}$.

We consider the spectral problem for the integrable system (2.5). Our general conjecture (1.10) applied to this case states that the spectrum of (2.5) on level $N$ is given by the Bethe anzatz equations

$$
\frac{\mathbb{A}\left(x_{i}-\frac{Q}{2}\right)}{\mathbb{A}\left(x_{i}+\frac{Q}{2}\right)} \prod_{j \neq i} \mathbb{S}\left(x_{i}-x_{j}\right)=q, \quad \text { for } \quad i=1, \ldots, N,
$$

where

$$
\mathbb{S}(x)=\frac{(x-b)\left(x-b^{-1}\right)(x+Q)}{(x+b)\left(x+b^{-1}\right)(x-Q)}, \quad \mathbb{A}(x)=\prod_{k=1}^{r}\left(x+i P_{k}\right) .
$$

Actually, we can formulate the statement more precisely and express the eigenvalues of the operators $\mathbf{I}_{k}$ in terms of Bethe roots. For example, the eigenvalues of the operator $\mathbf{I}_{2}$ are given by

$$
\mathbf{I}_{2} \sim \mathbf{I}_{2}^{\mathrm{vac}}+2 i \sqrt{r} \sum_{k=1}^{N} x_{k},
$$

where $\mathbf{I}_{2}^{\text {vac }}$ is an eigenvalue of the primary state $|\mathcal{P}\rangle$. Eigenvalues for other IM's are given by higher order power sum symmetric polynomials (more eigenvalues for the case $r=2$ can be found in [1]).

Let us comment on a local limit $q \rightarrow 1$. In this limit the Heisenberg part of the symmetry algebra decouples and the system (2.7) describes the spectrum of local IM's in the $\mathrm{W}_{r}$ algebra or qKdV $(r, 1)$ in our notations. First two IM's in this system have the form

$$
\mathbf{I}_{1}^{\text {local }}=L_{0}-\frac{c}{24}, \quad \mathbf{I}_{2}^{\text {local }}=W_{0} .
$$

On the other hand, the limit $q \rightarrow 1$ of the integral $\mathbf{I}_{2}$ from (2.5) is formally singular. In fact, the singularity is cancelled if one restricts the operator $\mathbf{I}_{2}$ to the purely $\mathrm{W}_{r}$ subspace 
(the set of states $|\lambda\rangle \in \pi_{\mathcal{P}}: a_{n}|\lambda\rangle=0$ for $n>0$ ). On this subspace

$$
\mathbf{I}_{2}^{\text {local }}=\frac{1}{\sqrt{\frac{r\left(r^{2}-4\right) Q^{2}}{2}+2(r-2)}} \mathbf{I}_{2} .
$$

The same is true for higher order integrals $\mathbf{I}_{k}$. We note also that for $r=1$ nothing remains from the symmetry algebra in the local limit. Technically, it is equivalent to the statement that BA equations (2.7) have no solutions for $r=1$ and $q=1$. We note also that the spectrum of $q \mathrm{KdV}(2,1)$ system was computed in [28] using the so called ODE/IM correspondence. Our equations are different from those in [28] (see discussion in [1]).

\section{The case $r=1$ and generic $p: \widehat{\mathfrak{g l}}(p)_{1}$ algebra}

In this case $\mathcal{A}(1, p)=0$. The remaining part of the symmetry algebra $\widehat{\mathfrak{g l}}(p)_{1}$ can be represented by $p$ complex fermions

$$
\psi^{a}(x)=\sum_{s} \psi_{s}^{a} e^{-i s x}, \quad \bar{\psi}^{a}(x)=\sum_{s} \bar{\psi}_{s}^{a} e^{-i s x}, \quad a=1, \ldots, p,
$$

with anticommutation relations

$$
\left\{\psi_{s}^{a}, \psi_{r}^{b}\right\}=\left\{\bar{\psi}_{s}^{a}, \bar{\psi}_{r}^{b}\right\}=0, \quad\left\{\psi_{s}^{a}, \bar{\psi}_{r}^{b}\right\}=\delta^{a, b} \delta_{r,-s} .
$$

Here we consider only Neveu-Schwarz sector, i.e. $s, r \in \mathbb{Z}+\frac{1}{2}$. The highest weight representation $\mathcal{F}$ of the fermionic algebra (3.2) is obtained in a usual way from the vacuum state $|0\rangle$ defined by $\psi_{s}^{a}|0\rangle=\bar{\psi}_{s}^{a}|0\rangle=0$ for $s>0$. This representation naturally decomposes into the direct sum $\mathcal{F}=\oplus \mathcal{F}_{l}$ where $\mathcal{F}_{l}$ is charge $l$ fermionic Fock module

$$
\mathcal{F}_{l}=\operatorname{Span}\left\{\prod_{a=1}^{p} \psi_{-s_{1}^{a}}^{a} \ldots \psi_{-s_{n_{a}}^{a}}^{a} \bar{\psi}_{-r_{1}^{a}}^{a} \ldots \bar{\psi}_{-r_{m_{a}}^{a}}^{a}|0\rangle: \sum_{a=1}^{p} n_{a}-\sum_{a=1}^{p} m_{a}=l\right\} .
$$

Each Fock module $\mathcal{F}_{l}$ forms an irreducible representation of $\widehat{\mathfrak{g l}}(p)_{1}$. They are all isomorphic to each other. For simplicity, we consider only $\mathcal{F}_{0}$.

According to our conjecture there exists an integrable system in $\mathcal{U}\left(\widehat{\mathfrak{g} l}(p)_{1}\right)$ which depends on $p$ external parameters $q_{1}, \ldots, q_{p}$ whose spectrum is described by (1.10). It would be convenient to introduce the other parameters $q$ and $\alpha_{1}, \ldots, \alpha_{p-1}$ by

$$
q_{1}=\frac{q}{\alpha_{1} \ldots \alpha_{p-1}}, \quad q_{2}=\alpha_{1}, \quad \ldots, \quad q_{p}=\alpha_{p-1} .
$$

Then the first two Integrals of Motion of our integrable system are (here :: stands for the

Wick ordering)

$$
\begin{aligned}
& \mathbf{I}_{1}=\frac{1}{2 \pi} \int_{0}^{2 \pi}: \bar{\psi}^{a} \partial \psi^{a}: d x, \\
& \mathbf{I}_{2}=\frac{1}{2 \pi} \int_{0}^{2 \pi}:\left(\frac{i\left(b-b^{-1}\right)}{2} \bar{\psi}^{a} \partial^{2} \psi^{a}+\frac{i Q}{2 p}:\left(\partial \psi^{a} \bar{\psi}^{a}+\partial \bar{\psi}^{a} \psi^{a}\right): \mathbf{H}: \bar{\psi}^{b} \psi^{b}:+\frac{i Q}{2 p}: \bar{\psi}^{a} \psi^{b}: \mathbf{D}^{a b}: \bar{\psi}^{b} \psi^{a}:\right): d x,
\end{aligned}
$$


where the Fourier symbols of the operators $\mathbf{H}$ and $\mathbf{D}^{a b}$ are

$$
\mathbf{H}(k)=\operatorname{sgn}(k), \quad \mathbf{D}^{a b}(k)=k \frac{1+\alpha_{a} \ldots \alpha_{b-1} q^{k}}{1-\alpha_{a} \ldots \alpha_{b-1} q^{k}} \quad \text { for } \quad a \leq b \quad \text { and } \quad \mathbf{D}^{b a}(k)=\mathbf{D}^{a b}(-k),
$$

and the sum over the repeated indexes is assumed. In principle, one can find higher IM's from the condition of mutual commutativity with $\mathbf{I}_{1}$ and $\mathbf{I}_{2}$, but their explicit form is more cumbersome.

The space $\mathcal{F}_{0}$ has natural gradings: the level $N$ and the fermionic numbers $\nu_{a}$ defined by

$$
N=\sum_{a=1}^{p}\left(\sum_{i=1}^{n_{a}} s_{i}^{a}+\sum_{j=1}^{m_{a}} r_{j}^{a}\right), \quad \nu_{a}=n_{a}-m_{a}
$$

Hence this space decomposes into the direct sum $\mathcal{F}_{0}=\oplus \mathcal{F}_{0}^{(N, \vec{\nu})}$ where

$$
\mathcal{F}_{0}^{(N, \vec{\nu})}=\operatorname{Span}\left\{\prod_{a=1}^{p} \psi_{-s_{1}^{a}}^{a} \ldots \psi_{-s_{n_{a}}^{a}}^{a} \bar{\psi}_{-r_{1}^{a}}^{a} \ldots \bar{\psi}_{-r_{m_{a}}^{a}}^{a}|0\rangle: \sum_{a=1}^{p}\left(\sum_{i=1}^{n_{a}} s_{i}^{a}+\sum_{j=1}^{m_{a}} r_{j}^{a}\right)=N, n_{a}-m_{a}=\nu_{a}\right\} .
$$

Clearly, the integrals $\mathbf{I}_{k}$ conserve the gradings. Therefore, our spectral problem decomposes into the collection of finite dimensional spectral problems on $\mathcal{F}_{0}^{(N, \vec{\nu})}$. We claim that the spectrum on $\mathcal{F}_{0}^{(N, \vec{\nu})}$ is governed by the BA equations (1.10) with

$$
A_{1}(x)=x, \quad A_{2}(x)=\cdots=A_{p}(x)=1, \quad N_{k}=N+\sum_{a=1}^{k-1} \nu_{a} .
$$

In particular, the spectrum of the integral $\mathbf{I}_{2}$ is given by the sum

$$
\mathbf{I}_{2} \sim \frac{2 i}{p} \sum_{j=1}^{N_{1}} x_{j}^{(1)}
$$

We note that in the limit $\left(q_{1}, \ldots, q_{p}\right) \rightarrow(0, \ldots, 0)$ the integral $\mathbf{I}_{2}$ coincides with the Hamiltonian of the spin Calogero model [21] written in a second quantized form (see [29]). One can easily see that the spectrum is degenerate in this limit. The reason for this degeneracy is well known [30]: the Integrals of Motion of the spin Calogero model commute with the Yangian $Y(\mathfrak{g l}(p))$. We were unable to find if there is a part of this symmetry which survives the perturbation (switching-on of the parameters $q_{k}$ ).

\section{The case $r=p=2$ : quantum SUSY KdV system}

Here, for simplicity, we consider only the local case $q_{1}=q_{2}=1$. According to our conjecture $\widehat{\mathfrak{g l}}(2)_{2}$ part of the algebra (1.6) decouples in this limit. Then we use the fact that $\mathcal{A}(2,2)=\mathrm{NSR}$, where NSR is a superconformal algebra (Neveu-Schwarz-Ramond algebra). This algebra is generated by currents $T(z)$ and $G(z)$ of spins 2 and $3 / 2$. Their components $L_{n}$ 's and $G_{r}$ 's satisfy the relations

$$
\begin{aligned}
& {\left[L_{n}, L_{m}\right]=(n-m) L_{n+m}+\frac{c}{8}\left(n^{3}-n\right) \delta_{n+m, 0},} \\
& \left\{G_{r}, G_{s}\right\}=2 L_{r+s}+\frac{1}{2} c\left(r^{2}-\frac{1}{4}\right) \delta_{r+s, 0}, \quad\left[L_{n}, G_{r}\right]=\left(\frac{1}{2} n-r\right) G_{n+r},
\end{aligned}
$$


where according to (1.3): $c=1+2 Q^{2}$. Below we will consider only Neveu-Schwarz sector, so the indexes $r$ and $s$ in (4.1) take half-integer values. The highest weight representation $\pi_{P}$ of (4.1) is defined by the vacuum state $|P\rangle$

$$
L_{n}|P\rangle=G_{r}|P\rangle=0 \quad \text { for } \quad n, r>0, \quad L_{0}|P\rangle=\Delta(P)|P\rangle,
$$

where $\Delta(P)=\frac{1}{2}\left(\frac{Q^{2}}{4}+P^{2}\right)$, and consists of the vectors of the form

$$
L_{-n_{1}} \ldots L_{-n_{k}} G_{-s_{1}} \ldots G_{-s_{l}}|P\rangle, \quad \text { where } \quad n_{1} \geq n_{2} \ldots, \quad s_{1}>s_{2}>\ldots
$$

The integrable system which appears in this case coincides with the quantum SUSY $\mathrm{KdV}$ system [31] whose local Integrals of Motion have the form ${ }^{1}$

$$
\mathbf{I}_{1}=\frac{1}{2 \pi} \int_{0}^{2 \pi} T d x, \quad \mathbf{I}_{3}=\frac{1}{2 \pi} \int_{0}^{2 \pi}\left(T^{2}+\frac{i}{4} G G^{\prime}\right) d x, \quad \ldots
$$

The spectral problem for the quantum SUSY KdV system was considered in [33, 34]. We suggest a different approach to the same problem. For us it will be important, that there is also a set of non-local Integrals of Motion of integer spin which commute with the local ones. First of them has the form

$$
\tilde{\mathbf{I}}_{1}=\frac{1}{2 \pi} \int_{0}^{2 \pi}\left(G \partial^{-1} G\right) d x
$$

The expressions for higher non-local integrals are known only in the classical limit [35], but there is no doubts that one can find their quantum analogs.

We claim that the spectrum of quantum SUSY KdV system in Neveu-Schwarz sector is governed by Bethe Anzatz equations (1.10) with $p=r=2, q_{1}=q_{2}=1$ and

$$
A_{1}(x)=x^{2}+P^{2}, \quad A_{2}(x)=1 .
$$

Exact expressions for the eigenvalues depend on the value of the level $N=\sum_{k} n_{k}+\sum_{r} s_{r}$ in (4.3) which can be either integer or half-integer. Namely, one has

- For integer $N$ we take $N_{1}=N_{2}=N$ in (1.10). Then the eigenvalues of the operator $\mathbf{I}_{3}+\frac{9}{16} i \tilde{\mathbf{I}}_{1}$ are given by

$$
\mathbf{I}_{3}+\frac{9}{16} i \tilde{\mathbf{I}}_{1} \sim \mathbf{I}_{3}^{\mathrm{vac}}(\Delta+N)+4 N \Delta+2 N(N-2)+6 \sum_{k=1}^{N}\left(x_{k}^{(1)}\right)^{2} .
$$

- For half-integer $N$ we take $N_{1}=N+\frac{1}{2}, N_{2}=N-\frac{1}{2}$ in (1.10), then

$$
\mathbf{I}_{3}-\frac{15}{16} i \tilde{\mathbf{I}}_{1} \sim \mathbf{I}_{3}^{\mathrm{vac}}(\Delta+N)+4\left(N+\frac{3}{2}\right) \Delta+2\left(N+\frac{3}{2}\right)\left(N-\frac{1}{2}\right)+6 \sum_{k=1}^{N+\frac{1}{2}}\left(x_{k}^{(1)}\right)^{2},
$$

here $\mathbf{I}_{3}^{\mathrm{vac}}(\Delta)$ is the eigenvalue of the primary state. We note here the phenomenon which was not present in the cases considered before. Namely, only special combinations of local and non-local IM's have eigenvalues which depend polynomially on Bethe roots. On lower levels we found that the eigenvalues of the local integral $\mathbf{I}_{3}$ are some rational symmetric functions of $x_{k}^{(1)}$, but their general form is not known.

\footnotetext{
${ }^{1}$ Here all the fields are assumed to be analytically regularized. See for example [32].
} 


\section{Conclusions}

In these notes we studied the spectrum of the local Integrals of Motion in the coset CFT (1.1). In fact, we noticed that it is quite natural to extend the coset algebra $\mathcal{A}(r, p)$ by $\widehat{\mathfrak{g l}}(p)_{r}$. We proposed that there exists the system of IM's in the universal enveloping of the extended algebra which depends on $p$ external parameters. We called this system qILW $(r, p)$ system and claimed that its spectrum is given by the Bethe anzatz equations (1.10). We have verified our conjecture for particular values of $r$ and $p$. Let us make a few comments which seem to be important.

1. It is now clear that the integrable system considered in this paper follows from certain $R$-matrix with parameters $q_{k}$ being twist parameters. It can be thought as an $R$-matrix for the Yangian $Y(\widehat{\mathfrak{g l}}(p))$ [36]. What is not known is the chain of arguments leading from this $R$-matrix to the Bethe equations (1.10). In other words we do not know how to develop the Bethe anzatz (algebraic, functional or whatever else) in this case.

2. As we explained in the introduction the chiral algebra description of the coset CFT (1.1) is too hard to analyze due to the presence of the currents with non-abelian braiding. It is believed that there exist local symmetry description of the same CFT. Such a description was found in $[37,38]$ for $r=2$. The corresponding chiral algebra is generated by $W$ current of spin 4 and can be realized in terms of three bosonic fields. One of the advantages of this description is that it is continuous in both parameters of the coset (1.1). In our approach one of the parameters of the coset (1.1) is necessarily an integer (we take $p$ to be integer and $n$ to be continuous). It would be interesting to find a way to generalize the Bethe anzatz equations (1.10) for non-integer values of $p$. We note that the spectrum of local IM's in three-field theory (also known as Fateev model) was computed in $[39,40]$ using ODE/IM correspondence. The relation between ODE/IM approach and ours is not clear.

\section{Acknowledgments}

A.L. thanks Sergei Lukyanov, Pierre Mathieu, Alexei Oblomkov, Andrei Zayakin and especially Mikhail Bershtein for interesting discussions. This research was done with the support of the Russian Scientific Foundation under the grant 14-12-01383. The work of M.A. was supported by the People Programme (Marie Curie Actions) of the European Union's Seventh Framework Programme under Grant Agreement No 317089 and by the 2013 Dynasty Foundation Grant. M.A. is very grateful to the Institute for Advanced Study (Princeton), where a part of this work was done, for hospitality.

Open Access. This article is distributed under the terms of the Creative Commons Attribution License (CC-BY 4.0), which permits any use, distribution and reproduction in any medium, provided the original author(s) and source are credited. 


\section{References}

[1] A.V. Litvinov, On spectrum of ILW hierarchy in conformal field theory, JHEP 11 (2013) 155 [arXiv: 1307.8094] [INSPIRE].

[2] P. Goddard, A. Kent and D.I. Olive, Virasoro algebras and coset space models, Phys. Lett. B 152 (1985) 88 [INSPIRE].

[3] C. Ahn, D. Bernard and A. LeClair, Fractional supersymmetries in perturbed coset CFTs and integrable soliton theory, Nucl. Phys. B 346 (1990) 409 [InSPIRE].

[4] A.B. Zamolodchikov, Higher order integrals of motion in two-dimensional models of the field theory with a broken conformal symmetry, JETP Lett. 46 (1987) 160 [INSPIRE].

[5] A.B. Zamolodchikov, Infinite additional symmetries in two-dimensional conformal quantum field theory, Theor. Math. Phys. 65 (1985) 1205 [INSPIRE].

[6] V.A. Fateev and A.B. Zamolodchikov, Conformal quantum field theory models in two-dimensions having $Z_{3}$ symmetry, Nucl. Phys. B 280 (1987) 644 [INSPIRE].

[7] V.A. Fateev and S.L. Lukyanov, The models of two-dimensional conformal quantum field theory with $Z_{n}$ symmetry, Int. J. Mod. Phys. A 3 (1988) 507 [InSPIRE].

[8] V.A. Fateev and A.B. Zamolodchikov, Representations of the algebra of 'parafermion currents' of spin 4/3 in two-dimensional conformal field theory. Minimal models and the tricritical potts $Z_{3}$ model, Theor. Math. Phys. 71 (1987) 451 [InSPIRE].

[9] A. LeClair, D. Nemeschansky and N.P. Warner, $S$ matrices for perturbed $N=2$ superconformal field theory from quantum groups, Nucl. Phys. B 390 (1993) 653 [hep-th/9206041] [INSPIRE].

[10] D. Gepner, New conformal field theories associated with Lie algebras and their partition functions, Nucl. Phys. B 290 (1987) 10 [InSPIRE].

[11] B.A. Kupershmidt and P. Mathieu, Quantum Korteweg-de Vries like equations and perturbed conformal field theories, Phys. Lett. B 227 (1989) 245 [INSPIRE].

[12] L.F. Alday, D. Gaiotto and Y. Tachikawa, Liouville correlation functions from four-dimensional gauge theories, Lett. Math. Phys. 91 (2010) 167 [arXiv:0906.3219] [INSPIRE].

[13] V. Belavin and B. Feigin, Super Liouville conformal blocks from $N=2 \mathrm{SU}(2)$ quiver gauge theories, JHEP 07 (2011) 079 [arXiv: 1105.5800] [INSPIRE].

[14] T. Nishioka and Y. Tachikawa, Central charges of para-Liouville and Toda theories from M-5-branes, Phys. Rev. D 84 (2011) 046009 [arXiv: 1106.1172] [INSPIRE].

[15] A. Belavin, V. Belavin and M. Bershtein, Instantons and 2d superconformal field theory, JHEP 09 (2011) 117 [arXiv: 1106.4001] [INSPIRE].

[16] G. Bonelli, K. Maruyoshi and A. Tanzini, Instantons on ALE spaces and super Liouville conformal field theories, JHEP 08 (2011) 056 [arXiv:1106.2505] [INSPIRE].

[17] M.N. Alfimov and G.M. Tarnopolsky, Parafermionic Liouville field theory and instantons on ALE spaces, JHEP 02 (2012) 036 [arXiv:1110.5628] [INSPIRE].

[18] M.N. Alfimov, A.A. Belavin and G.M. Tarnopolsky, Coset conformal field theory and instanton counting on $C^{2} / Z_{p}$, JHEP 08 (2013) 134 [arXiv:1306.3938] [INSPIRE]. 
[19] A.A. Belavin, M.A. Bershtein, B.L. Feigin, A.V. Litvinov and G.M. Tarnopolsky, Instanton moduli spaces and bases in coset conformal field theory,

Commun. Math. Phys. 319 (2013) 269 [arXiv:1111.2803] [InSPIRE].

[20] A.A. Belavin, M.A. Bershtein and G.M. Tarnopolsky, Bases in coset conformal field theory from AGT correspondence and Macdonald polynomials at the roots of unity, JHEP 03 (2013) 019 [arXiv:1211.2788] [INSPIRE].

[21] Z.N.C. Ha and F.D.M. Haldane, Models with inverse-square exchange, Phys. Rev. B 46 (1992) 9359 [inSPIRE].

[22] D. Uglov, Yangian Gelfand-Zetlin bases, $\mathrm{gl}_{N}-$ Jack polynomials and computation of dynamical correlation functions in the spin Calogero-Sutherland model, Commun. Math. Phys. 193 (1998) 663 [hep-th/9702020] [INSPIRE].

[23] N.A. Nekrasov and S.L. Shatashvili, Supersymmetric vacua and Bethe ansatz, Nucl. Phys. Proc. Suppl. 192-193 (2009) 91 [arXiv:0901.4744] [INSPIRE].

[24] A. Okounkov and R. Pandharipande, The quantum differential equation of the Hilbert scheme of points in the plane, Transform. Groups 15 (2010) 965 [arXiv:0906.3587].

[25] D. Maulik and A. Oblomkov, Quantum cohomology of the Hilbert scheme of points on $A_{n}$-resolutions, J. Am. Math. Soc. 22 (2009) 1055 [arXiv:0802.2737].

[26] G. Bonelli, A. Sciarappa, A. Tanzini and P. Vasko, Six-dimensional supersymmetric gauge theories, quantum cohomology of instanton moduli spaces and $\mathrm{gl}(N)$ quantum intermediate long wave hydrodynamics, JHEP 07 (2014) 141 [arXiv: 1403.6454] [INSPIRE].

[27] S. Nawata, Givental J-functions, quantum integrable systems, AGT relation with surface operator, arXiv: 1408.4132 [INSPIRE].

[28] V.V. Bazhanov, S.L. Lukyanov and A.B. Zamolodchikov, Higher level eigenvalues of $Q$ operators and Schrödinger equation, Adv. Theor. Math. Phys. 7 (2004) 711 [hep-th/0307108] [INSPIRE].

[29] I. Aniceto and A. Jevicki, Notes on collective field theory of matrix and spin Calogero models, J. Phys. A 39 (2006) 12765 [hep-th/0607152] [InSPIRE].

[30] D. Bernard, M. Gaudin, F.D.M. Haldane and V. Pasquier, Yang-Baxter equation in spin chains with long range interactions, J. Phys. A 26 (1993) 5219 [hep-th/9301084] [INSPIRE].

[31] P. Mathieu, Integrability of perturbed superconformal minimal models, Nucl. Phys. B 336 (1990) 338 [inSPIRE].

[32] V.V. Bazhanov, S.L. Lukyanov and A.B. Zamolodchikov, Integrable structure of conformal field theory, quantum KdV theory and thermodynamic Bethe ansatz, Commun. Math. Phys. 177 (1996) 381 [hep-th/9412229] [INSPIRE].

[33] P.P. Kulish and A.M. Zeitlin, Superconformal field theory and SUSY N $=1$ KdV hierarchy I: vertex operators and Yang-Baxter equation, Phys. Lett. B 597 (2004) 229 [hep-th/0407154] [INSPIRE].

[34] P.P. Kulish and A.M. Zeitlin, Superconformal field theory and SUSY N $=1$ KDV hierarchy II: the Q-operator, Nucl. Phys. B 709 (2005) 578 [hep-th/0501019] [INSPIRE].

[35] S. Andrea, A. Restuccia and A. Sotomayor, Infinite sequence of new conserved quantities for $N=1 S K d V$ and the supersymmetric cohomology, arXiv:0811.1246 [INSPIRE]. 
[36] D. Maulik and A. Okounkov, Quantum groups and quantum cohomology, arXiv:1211.1287 [INSPIRE].

[37] V.A. Fateev, The $\sigma$-model (dual) representation for a two-parameter family of integrable quantum field theories, Nucl. Phys. B 473 (1996) 509 [INSPIRE].

[38] B.L. Feigin and A.M. Semikhatov, The affine $\hat{\mathrm{sl}}(2) \oplus \hat{\mathrm{sl}}(2) / \hat{\mathrm{sl}}(2)$ coset theory as a Hamiltonian reduction of $\hat{D}(2 \mid 1: \alpha)$, Nucl. Phys. B 610 (2001) 489 [hep-th/0102078] [InSPIRE].

[39] S.L. Lukyanov, ODE/IM correspondence for the Fateev model, JHEP 12 (2013) 012 [arXiv: 1303.2566] [INSPIRE].

[40] V.V. Bazhanov and S.L. Lukyanov, Integrable structure of quantum field theory: classical flat connections versus quantum stationary states, JHEP 09 (2014) 147 [arXiv:1310.4390] [INSPIRE]. 